\title{
An experimental investigation of how addressee feedback affects co-speech gestures accompanying speakers' responses
}

\author{
Judith Holler ${ }^{\mathrm{a}, \mathrm{b}, *}$, Katie Wilkin ${ }^{\mathrm{b}}$ \\ ${ }^{a}$ Max Planck Institute for Psycholinguistics, Wundtlaan 1, 6525 XD Nijmegen, The Netherlands \\ ${ }^{\mathrm{b}}$ School of Psychological Sciences, University of Manchester, Coupland Building 1, Oxford Road, Manchester M13 9PL, UK
}

\section{A R T I C L E I N F O}

\section{Article history:}

Received 20 May 2011

Received in revised form 1 August 2011

Accepted 2 August 2011

Available online 3 September 2011

\section{Keywords:}

Co-speech gesture

Addressee feedback

Recipient design

Gaze

Verbal deixis

\begin{abstract}
A B S T R A C T
There is evidence that co-speech gestures communicate information to addressees and that they are often communicatively intended. However, we still know comparatively little about the role of gestures in the actual process of communication. The present study offers a systematic investigation of speakers' gesture use before and after addressee feedback. The findings show that when speakers responded to addressees' feedback gesture rate remained constant when this feedback encouraged clarification, elaboration or correction. However, speakers gestured proportionally less often after feedback when providing confirmatory responses. That is, speakers may not be drawing on gesture in response to addressee feedback per se, but particularly with responses that enhance addressees' understanding. Further, the large majority of speakers' gestures changed in their form. They tended to be more precise, larger, or more visually prominent after feedback. Some changes in gesture viewpoint were also observed. In addition, we found that speakers used deixis in speech and gaze to increase the salience of gestures occurring in response to feedback. Speakers appear to conceive of gesture as a useful modality in redesigning utterances to make them more accessible to addressees. The findings further our understanding of recipient design and co-speech gestures in face-to-face dialogue.
\end{abstract}

(c) 2011 Elsevier B.V. All rights reserved.

\section{Introduction}

Communication is a collaborative, joint activity and requires the close coordination of two or more individuals (Clark, 1996; Garrod and Anderson, 1987). Even when one participant tells a longer story, anecdote, joke or such like, addressees are still active contributors; the feedback they provide is a crucial component in the process of communication. Allwood and Ahlsén (1999) name 'feedback' as one of the three most important prerequisites to interactively manage communication (next to turn-taking and sequencing). And indeed, research has shown that verbal feedback enhances speakers' communicative performance, for example in terms of efficiency (Krauss and Weinheimer, 1966) and the quality of storytelling (Bavelas et al., 2000). Likewise, it has been shown that the opportunity to provide feedback improves recipients' understanding of communicative messages (Kraut et al., 1982; Schober and Clark, 1989).

However, face-to-face dialogue does not just consist of verbal communication, of course. Co-speech gestures play an important part in it (Bavelas and Chovil, 2000; Clark, 1996; Kendon, 2004; McNeill, 1992). Although their role in interaction and communication has received quite some attention in recent decades, not much is known about co-speech gestures in the context of addressee feedback.

\footnotetext{
* Corresponding author at: Max Planck Institute for Psycholinguistics, 6525 XD Nijmegen, The Netherlands. Tel.: +31 24 3521331; fax: +31 243521213. E-mail address: Judith.holler@mpi.nl (J. Holler).
} 
Co-speech gestures come in many forms, well known ones being pointing gestures and iconic depictions of aspects closely related to the content of the accompanying speech (McNeill, 1992). They are abundant in everyday talk, and a striking cross-cultural phenomenon (Kita, 2003). For quite some time, the one question that appeared to preoccupy many researchers in the field of gesture concerned their global function (i.e., inter- or intrapersonal). Nowadays, there is plenty of evidence that co-speech gestures appear to fulfil both speaker-oriented (e.g., Hostetter et al., 2007; Kita and Davies, 2009; Morsella and Krauss, 2004; Pine et al., 2010) and addressee-oriented functions (e.g., Alibali et al., 2001; Bavelas et al., 2008; Holler and Beattie, 2003b; Jacobs and Garnham, 2007).

Studies exploring the communicativeness of co-speech gestures and the extent to which they are recipient designed (Garfinkel, 1967; Sacks et al., 1974) have manipulated a range of social context variables. For example, participants have been asked to communicate in conditions in which speakers knew that addressees could or could not see their gestures, with the predominant finding that speakers gestured more when they knew their gestures could be seen (Alibali et al., 2001; Bavelas et al., 2002, 2008; Mol et al., 2009). Other studies have found evidence for recipient design in gesture by manipulating addressee location leading to speakers adjusting where and how the gestures were performed in gesture space (Furuyama, 2000; Özyürek, 2002). In addition, studies have shown that when gesturing, speakers take into account the knowledge they mutually share with their addressees, with effects on gesture rate, the semantic information gesturally encoded, and the physical form of gestures (Gerwing and Bavelas, 2004; Holler, 2010; Holler and Stevens, 2007; Holler and Wilkin, 2009; Parrill, 2010). However, all of these studies have taken a rather global approach by comparing overall gesture rates or form changes encouraged by the experimental manipulations (such as visibility, spatial location or mutually shared knowledge) rather than by addressee-related behaviours.

However, some studies have focused on the topic of gesture and addressee feedback, from a variety of different perspectives. One perspective is that of gestures constituting addressee feedback. This includes Tabensky's (2001) descriptive analysis of how speakers rephrase their interlocutors' gestures - here the gestures provide feedback to the addressee by offering a new or slightly different interpretation of what they relayed earlier. Within this same group, we find studies that have focused on a related yet different aspect, namely, interlocutors reproducing the same gesture as the previous speaker, also referred to as gesture mimicry (de Fornel, 1992; Holler and Wilkin, 2011; Kimbara, 2006, 2008). For example, Holler and Wilkin (2011) provided an overview of how, in the context of referential communication, mimicked gestures can provide important feedback for the preceding speaker about the comprehensibility of the message, thus playing a role in the process of grounding (Clark and Brennan, 1991). This is in line with findings from a study by Clark and Krych (2004) who examined the use of gestures performed on objects within a shared workspace. Participants trying to follow instructions given by another used these gestures to signal their understanding or problems in understanding and to request further information (and instructors gazed at these gestures to monitor their addressees' understanding). Again, the gestural feedback was thus used as an important source of information that facilitated the process of grounding.

Another group of studies has focused on gestures for eliciting addressee feedback (Bavelas et al., 1992, 1995). More specifically, these gestures have been termed 'seeking gestures' as they tend to be used to seek for help (e.g., in the context of searching for a word), to seek agreement (to be paraphrased as 'don't you think/agree?') or to seek evidence that the interlocutor has understood what has been said (to be paraphrased as 'you know/understand?'). These gestures form part of a larger class of gestures called 'interactive gestures' (Bavelas et al., 1992, 1995), which serve the function of regulating social interaction by making reference to, and thus including, the addressee in the interaction. Bavelas et al. (1995) showed that these postulated interactive functions, including the gestural seeking of addressee feedback, can be empirically evidenced on the basis of the addressees' responses.

A last group of studies can be summarised as focusing on gestures in response to addressee feedback. Using different approaches, these studies have explored the way in which addressee feedback influences both the occurrence as well as the form of co-speech gestures. Jacobs and Garnham (2007) showed that speakers gesture at a lower rate when their addressees seemed less attentive (due to providing less feedback cues as they were distracted by a secondary task while listening). Similarly, Beattie and Aboudan (1994) manipulated the social context during story telling and found that speakers gestured at a higher rate with addressees who asked questions and provided other types of feedback as compared to addressees who did not engage in any verbal exchange. They also found that gestures had a longer duration when talking to an addressee who provided feedback than one who did not.

However, a question that has received very little attention so far is how speakers adjust their gestures in response to specific types of addressee feedback (rather than a general impression of attentiveness or inattentiveness due to an overall higher or lower degree of feedback, or an impression of the degree of involvement, based on the number of questions asked). Exceptions are two qualitative studies by Streeck $(1993,1994)$ in which he describes ways in which recipients can act as coauthors of speakers' gestures. Streeck's analyses show that speakers are sensitive to their addressees' behaviour, in particular the recipient's gaze direction. In one of the examples he provides, the recipient's gaze returns to the speaker after a period of gaze aversion; at this point, she repeats her previous gesture and, in addition, creates a more visible gestural representation which is also more representative (or iconic) in nature (Streeck, 1994). Another example Streeck (1994) provides stems from a word search context. The speaker is trying to recall a particular word accompanied by a gesture referring to the concept in question. She thus elicits a guess from the addressee, but in response to this guess being wrong, adapts her gesture so that it becomes a more specific representation of the concept for which she is trying to retrieve the lexical label.

Another exception is an example provided by Kendon (2004), taken from a conversation between two individuals (A and B). In this example, A refers to certain individuals having made an area greasy, which he accompanies with a related gestural 
representation (depicting an area being covered in grease). His interlocutor, B, signals that he did not understand A's utterance ('they made what?'), in response to which A repeats it, including the previous gesture. This is followed by a second request for clarification by $B$, this time indicating more precisely which aspect of the message he did not understand (i.e., the word 'greasy'). The second repair A undertakes, however, differs from the previous one - this time, he changes the form of his gesture when repeating the word 'greasy'. Furthermore, B then does understand and repeats the word 'greasy' himself to signal this. A then also repeats the word 'greasy' once more to confirm that B has understood correctly, but this time without a gesture. Kendon (2004) emphasises that the important point to note here is that, in repeating and revising his utterance, A includes both speech and gesture. This, Kendon states, is evidence that speakers consider both modalities as partners on an equal footing. He refers to utterances as gesture-speech ensembles in which speakers are free to re-design the interplay of the two modalities as appears most appropriate with respect to the conversational context.

The descriptive analyses by Streeck $(1993,1994)$ and Kendon (2004) nicely demonstrate how individual gestures (or gesture-speech ensembles) are used in conversation to respond to addressees' feedback behaviour. However, systematic comparisons of how addressee feedback influences speakers' co-speech gestures have remained at a global level, with the general finding that more interaction and more perceived attentiveness leads to a higher gesture rate (Beattie and Aboudan, 1994; Bavelas et al., 2008; Jacobs and Garnham, 2007). This leaves important questions unanswered. What are the prevalent patterns for gestural responses to addressee feedback? Kendon (2004) observed three different ways in which speaker A responded to his addressee's feedback - he repeated the same gesture (albeit with a slightly smaller amplitude), he used a different gesture replacing the earlier one, and with his third response he used no gesture at all. Do speakers generally draw upon the gestural modality when refashioning utterances in response to addressee feedback? If so, does the type of feedback play a role here, as Kendon suggested (non-understanding of the entire message versus a particular word)? Considering the form of gestures, is gestural repetition the predominant response to addressee feedback, or do we find evidence that speakers also make their gestures more communicative sometimes? If so, how common are repetition or change in response to feedback? When the same gesture is repeated in response to addressee feedback, this is strong evidence that gesture and speech form a tightly integrated unit (a gesture-speech ensemble, in Kendon's words). However, theoretically, the gesture could still occur as some form of by-product of repeating the verbal component of the utterance. Much stronger evidence for gestures being part of the speaker's attempt to create a more informative utterance in response to their addressee's feedback (i.e., an utterance in which the gesture is clearly recipient-designed) is when we observe change - and not just any change, but change towards gestures becoming more communicative after feedback.

In the present study we aim to answer the above questions by providing both an analysis of gesture rate and the qualitative form of gestures before and after addressee feedback. In order to allow for a systematic comparison, we asked participants to provide narratives to a confederate addressee who provided scripted feedback at certain points during these narratives. This way, we could base our analyses on gestures referring to the same semantic events told by different speakers - thus avoiding the potential problem of differences in the topic of talk or in the types of feedback different addressees may have provided to the individual speakers. Further, we needed a controlled stimulus to be able to script feedback that tapped the semantic aspects that speakers tended to include in their narratives (which we did based on narratives obtained in an earlier experiment using the same stimulus).

Addressee feedback takes on many forms. It includes continued gaze as an expression of willingness and ability to communicate, nonverbal signals such as head shakes/nods (Kopp et al., 2008), small verbal morphemes such as 'yes', 'ok', 'uhu', 'mm' and so forth (which have been termed back-channel responses (Yngve, 1970), continuers (Schegloff, 1982) or acknowledgements (Clark and Schäfer, 1987) amongst others). In addition to small responses such as these, interlocutors also provide longer interjections, typically of the length of a clause or a short sentence (and sometimes longer than that), such as 'how far is it from Huddersfield to Coventry?' (Clark and Schäfer, 1987). Thus, participants in dialogue employ a multi-modal system of contributions, including both positive and negative signals of understanding and contributions of varying sizes. In the present study, the addressee was free to provide the whole range of feedback types that we encounter in everyday conversation (such as the ones mentioned above). She was asked to signal her understanding or non-understanding spontaneously, except that for the larger sized feedback contributions questions were scripted (e.g., 'he did what, sorry?').

As mentioned above, we compared speakers' gesture rate before and after feedback, and the qualitative nature of corresponding gestures (i.e., gestures referring to the same semantic event before and after feedback). With regard to gesture rate, we were interested in seeing to what extent speakers consider gesture as a crucial component of their response to addressee feedback. It could be that the predominant response is a purely verbal one that involves repetition of those aspects of the previous message that the addressee signals not to have understood. On the other hand, if co-speech gestures play an important communicative role in refashioning utterances in response to addressee feedback (as postulated by Kendon, 2004), speakers should continue to use gesture at the same or even a higher rate after addressee feedback.

Further, it is evident that when responding to addressees signalling problems in understanding, speakers change the form of their verbal contribution, such as by using louder or more carefully articulated speech (rarely do they repeat a message in exactly the same form as before, unless, perhaps, a source of noise prevented the addressee from hearing properly and this source has vanished). If speakers employ co-speech gestures as part of their recipient-designed responses to addressees, we would expect to also see differences in the gestures' qualitative form before and after feedback, making the gesture more communicative in some way. We begin to explore this issue here by focusing on four different dimensions, which have all been shown to be related to the communicativeness of co-speech gestures in previous research: (i) gesture precision (gestures associated with a need for greater communicativeness tend to be more precise; Gerwing and Bavelas, 2004), 
(ii) gesture size (more communicative gestures tend to be larger; Bavelas et al., 2008; Beattie and Shovelton, 2005; Enfield et al., 2007; Mol et al., 2009); (iii) gesture space (more communicative gestures tend to be performed in more visually prominent areas of gesture space; Streeck, 1994), and (iv) gesture viewpoint (character viewpoint gestures have been associated with greater communicative power than observer viewpoint gestures, Beattie and Shovelton, 2002). If co-speech gestures are recipient-designed in response to addressee feedback, we would expect them to show signs of increased communicativeness on all or some of these four dimensions. We therefore predicted that, on the whole, gestures after feedback would be more precise in their form, larger, performed in more visually prominent areas of gesture space, or that they would show a shift towards being performed from a more communicative gestural viewpoint or perspective.

Finally, we also explored the interplay between gestures and concurrent signals in other modalities increasing the communicativeness of the gestures, namely speech and gaze. More precisely, we focused on elements of deixis expressed in these modalities intended to draw attention to the gestures. Speakers have been shown to overtly integrate their iconic gestures into their verbal utterances to make their communicative relevance more explicit by employing verbal deictic markers such as 'it was this big' or 'it went round like that' (Slama-Cazacu, 1976; Goodwin, 1986). Also, speakers shift their gaze towards their own gestures to overtly mark their communicative relevance (Streeck, 1993; Goodwin, 1986). That is, speakers 'orient to their gestures as they are making them. Through their orientation, they can communicate to their interlocutors that the gesture is a legitimate object of attention. Speakers, in this fashion, establish their gestures as significant acts of communication' (Streeck, 1994:239). Moreover, recipients appear to pick up on this communicative cue as they direct their own gaze towards the speaker's gesture in response to these gaze shifts (Gullberg and Holmqvist, 1999, 2006). Thus, deictic cues in concurrent modalities can indicate an increase in the communicative relevance of individual gestures. If gestures are used to provide recipient-designed responses to addressee feedback, we would expect that speakers will use more of these deictic markers (or orientation cues) towards gesture in speech and in gaze after addressee feedback.

In sum, 'a speaker has to ensure that his utterances are constructed so that it can be expected that the listener can actually retrieve its original communicative intention. This holds not only for linguistic communication but also for non-linguistic communication of any kind' (De Ruiter et al., 2010:72). In other words, speakers ought to make sure that both the verbal and nonverbal components of their utterances are recipient-designed. We therefore expect speakers to comply with the Gricean maxim of quality, which predicts that speakers make their contributions as informative as required - and thus produce gestures that target the need for increased communicativeness expressed by the addressee's feedback. Here, we focus on two complementary measures, a purely quantitative one (gesture occurrence) and a form-based one, to provide us with a first glimpse of how speakers respond gesturally to addressee feedback in the context of narrative telling.

\section{Method}

\subsection{Participants}

Thirty-seven participants from the student population of the University of Manchester (UK) took part in the experiment. Of these, nine were excluded on the basis of manipulation checks (see section 2.2.2), thus reducing the total number of participants included in the analyses to 28. All participants were female native English speakers (age: $M=20.00$ yrs, $S D=2.33 \mathrm{yrs}) .{ }^{1}$ Participants were compensated financially or with course credits.

\subsection{Materials}

\subsubsection{Stimulus material}

The stimulus consisted of a $7 \mathrm{~min}$ long video recording extracted from a longer video from the German children television series 'Neues aus Uhlenbusch', episode 'Grosser kleiner Heiner' (permission for use in the experiment was obtained from the copyright holder ZDF). The stimulus video depicted a range of adult and child characters carrying out everyday activities such as grocery shopping, cycling through fields and children playing in a hay barn. The story told in the video had a clear plot with a beginning, middle and end. It was played without the sound but was still easily understandable (all participants told the story coherently and with a clear storyline).

\subsubsection{Scripted feedback}

In order to tailor the scripted feedback to appropriate aspects of the storyline speakers would be telling we consulted a corpus collected earlier (Holler and Wilkin, 2009) consisting of narratives based on the same stimuli as used in the present study. It was thus possible to identify those elements of the story that had a high likelihood of being mentioned by each speaker (resulting in four target scenes ${ }^{2}$ ). In scripting the feedback related to these selected points in the story, we made sure that the form of the feedback was representative of the kind of feedback addressees typically provided in the corpus we

\footnotetext{
${ }^{1}$ Due to potential gender differences when interacting with the female confederate, we decided to keep the gender pairing constant in this study. We opted for female rather than male speakers because the students enrolled in the psychology program at Manchester are almost exclusively female.

${ }^{2}$ Note that two more scenes were originally identified but not included in the current analysis as the scripted feedback delivered in these cases was purely nonverbal (a gesture used to signal understanding, and furrowed eyebrows indicating non-understanding, both performed without speech, during the speaker's turn) and did not result in explicit narrator responses.
} 
Table 1

Overview of target scenes and corresponding addressee feedback.

\begin{tabular}{|c|c|c|}
\hline Target scene & Feedback type & Form of feedback \\
\hline $\begin{array}{l}\text { Scene 1: One of the main characters almost gets } \\
\text { knocked over by a car }\end{array}$ & $\begin{array}{l}\text { Request for further information due } \\
\text { to lack of clarity/detail }\end{array}$ & $\begin{array}{l}\text { 'How close does it (the car) } \\
\text { get to him (the man)?' }\end{array}$ \\
\hline $\begin{array}{l}\text { Scene 2: The group of boys featured throughout } \\
\text { the story jump from an upper level in a hay } \\
\text { barn down into some hay below }\end{array}$ & $\begin{array}{l}\text { Expression of non-understanding - } \\
\text { provision of incorrect interpretation }\end{array}$ & $\begin{array}{l}\text { 'So you mean they (the boys) } \\
\text { jump out of the barn?' }\end{array}$ \\
\hline $\begin{array}{l}\text { Scene } 3 \text { : One of the main characters pokes his } \\
\text { head out of a gap in the barn roof and } \\
\text { overlooks the inner court of the barn }\end{array}$ & $\begin{array}{l}\text { Expression of non-understanding - } \\
\text { request for speaker to repeat or } \\
\text { clarify what she said }\end{array}$ & 'He does what, sorry?' \\
\hline $\begin{array}{l}\text { Scene } 4 \text { : The group of boys run out of the barn } \\
\text { and hide behind some crops to escape the } \\
\text { barn owner }\end{array}$ & $\begin{array}{l}\text { Seeking confirmation that understanding } \\
\text { is correct - provision of correct interpretation }\end{array}$ & $\begin{array}{l}\text { 'So they (the boys) all manage } \\
\text { to get out and hide?' }\end{array}$ \\
\hline
\end{tabular}

collected earlier (i.e., similar in terms of the semantic aspects tapped by the addressee's feedback, the level of semantic detail in those questions, and their grammatical form). All of the four feedback instances were questions, two of which can be classed as polar questions (offering a proposition that is either true or false; yes/no question; Scenes 2 and 4 ) and two as content questions (part of proposition addressed is presupposed and the question targets the remaining element; typically WH-questions in the English language; Scenes 1 and 3) (Stivers and Enfield, 2010). In terms of the social actions these questions initiate, they fall into the categories proposed by Stivers and Enfield (2010) as follows: request for information (Scene 1), other-initiation of repair (Scene 3), and request for confirmation (Scenes 2 and 4) (Table 1).

In addition, the female confederate who delivered the feedback (KW) was a member of the research team and thus well familiar with the content of the video and the scripted feedback. She was asked to combine consistency in feedback with acting naturally and as spontaneously as possible, despite the scripted nature of the feedback. This means that although the feedback occurred at predetermined points and in a predetermined form, it was left to the confederate to deviate from this script where it felt appropriate depending on the conversational context. This concerned the spontaneous production of cospeech gestures together with the scripted verbal feedback, although this was rather consistent across trials. For example, she tended to use a gesture depicting the path of the jumping movement for Scene 2, and a gesture depicting the characters running out and ducking down with the verbal feedback for Scene 4 . Scene 3 was never accompanied by a gesture. The feedback for Scene 1 was accompanied by a gesture on some of the trials (6/16); however, this slight variation seems to not have affected the speakers' gestures in any systematic way, as for three out of the six cases, the gesture rate for Scene 1 decreased after the addressee's feedback, while for the other three it increased. Also, these six speakers' average gesture rate (.17) was not significantly different from that of the remaining participants (.16), $U=51.50, n^{1}=6, n^{2}=19, p=.733$.

It was also up to the confederate to decide whether to deliver the feedback or not. For example, in some cases it did not appear appropriate to provide the feedback as the way the story was narrated would have required a very abrupt interruption from the addressee, or, due to the speed of talking, the speaker had already moved on from the relevant part of the story. In some other cases, the way in which the event was relayed would have created a pragmatically odd situation following the feedback, for example, if the aspect the feedback addressed had already been mentioned (such as a speaker describing, out of their own accord, how close the car got to the man). Similarly, if a speaker did not refer to a certain event or a part of it (such as the fact that the children ran out of the barn), then the addressee did not refer to the respective scene with the scripted feedback (in this case, tapping the act of running out) since a naïve interlocutor could not know about this aspect, but, instead, she enquired about the remaining aspects (e.g., the act of hiding only) or not at all if the entire event had not been mentioned.

More generally, the confederate was also free to vary her behaviour spontaneously to adapt to the communicative style of the participant. This means that the addressee's feedback might have been delivered slightly differently in terms of tone, emphasis and so forth when speaking to a loud, out-going person compared to a quieter and more timid speaker. The confederate was also free to ask any additional questions, make comments, laugh, or do anything else that they felt was necessary and appropriate to create a dynamic, naturalistic conversation in each case. Further, we did not prescribe the confederate to try to control her smiles/facial expressions, body posture, how they came across personally and so forth. Such factors, we decided, would run the risk of creating a forced or robotic impression and should remain dependent on the dynamics of the conversation and the social relationship between the interlocutors. That is, the interactions might have varied on these dimensions and, due the complexity of social interactions, we were not able to measure and quantify this variation. However, due to the fact that our analyses are based exclusively on within-participants measures, potential differences between dyads do not affect the individual before- and after feedback measures that we compared.

In addition, two judges looked through the entire set of video recordings to check that the interaction, as well as the way in which the feedback was delivered, seemed natural and consistent (in terms of the scripted aspects) across participants. Because it is difficult to develop formal criteria according to which 'natural behaviour' can be measured, the judges based their decisions on their personal perception. They agreed that the first seven trials should be excluded (due to this being the initial trials, the confederate seemed a little too concentrated on their own behaviour and thus less natural than in the remainder of trials. Trials 11 and 35 were excluded for similar reasons.); note that these trials were excluded before the analysis was carried out. 


\subsubsection{Questionnaires}

Participants completed a questionnaire designed to evaluate the cooperativeness of the addressee, the addressee's feedback and the quality of the communication. The questionnaire consisted of five questions, each to be answered on a 7-point Likert scale (ranging from 'not at all' to 'very much'). The questions were as follows: (1) 'How cooperative did you perceive the listener to be during the descriptions you provided?', (2) 'Once you had finished the story description, to what extent did you feel the listener had understood the story as you intended to convey it?, (3) How would you rate the quality of the listener's feedback?', (4) 'To what extent did you feel the listener's feedback tapped aspects that were relevant to understanding your description of the story?', (5) 'To what extent did the listener's feedback help you to communicate a. successfully, b. clearly, c. efficiently?'. A sixth, open-ended question was included at the end, (6) 'Please note down anything else you would like to comment on with respect to the listener's communicational behaviour'.

The questionnaire was aimed to serve as an indication as to how natural the participants experienced the confederate's behaviour. Participants in everyday conversation behave cooperatively by providing relevant information, the right amount of information, by communicating efficiently and by signalling problems in understanding if they occur (Clark, 1996; Grice, 1975). The participants' questionnaire evaluations were thus intended to indicate whether participants found the addressee's behaviour unnatural, thus interfering with the quality of their communication, and whether they noticed that the addressee was in fact a confederate (we did not want to ask explicitly whether participants had noticed that the addressee was a confederate as it bears the risk of participants answering 'yes' to such questions even if they did not, thinking that they should have noticed). The mean ratings for the five questions can be found in (Table 2).

These scores were interpreted as reflecting that participants experienced their addressee as cooperative and their communication as successful. Furthermore, none of the participants included comments indicating that they assumed the addressee to be a confederate or odd in their behaviour; quite the contrary: all of their responses were positive, consisting of comments such as: 'very encouraging and attentive, felt like she was really understanding what I was saying', 'asked helpful questions which showed me that I was successful in communicating the story to her', 'seemed very engaged and interested', 'clarified unclear points with questions, which was very helpful', 'was very attentive and tried to take in as much as possible', 'asked relevant questions, helped me to clarify my description', 'behaviour as expected from someone listening to a description', and so forth.

\subsubsection{Ethics documents and participant screening}

In addition, we provided all participants with an information sheet and consent forms. Participants were also asked to confirm, in writing, that they were native English speakers and that they had not been diagnosed with any language impairments.

\subsection{Procedure}

Participants met the researcher in the foyer of the psychology department. A confederate participant (female) joined them at this meeting point and pretended not to be familiar with the researcher (nor the experiment). The researcher introduced herself to the two participants and them to each other before she led them to the observation laboratory.

The experimental set-up included two low-seated chairs (without arm rests) placed opposite each other at a comfortable conversational distance, separated by a coffee table. The confederate chose the same seat during all trials. Both participants were asked to read through the information sheet, whether they had any further questions (which were clarified if they did), and for their consent before the study commenced. Participant then watched a video (section 2.2.1), the content of which they was asked to narrate to the 'other participant'. The confederate-addressee was instructed to listen carefully and to signal her understanding or non-understanding in any way she felt was necessary in order to arrive at a full understanding of the narrative (however, unknown to the participant, in addition to this, she was required to provide the scripted feedback, see section 2.2.2). To increase the speaker's communicative motivation, they were told that the addressee would be asked to answer questions about the content of the story at the end of the experiment.

Once the participant had completed their narrative, both participant and confederate were lead to separate tables to complete their questionnaire (section 2.2.3) to allow for sufficient privacy when evaluating the partner's behaviour (the confederate addressee pretended to also be completing the questionnaires). Following this, participants were debriefed and, again, asked for their written consent allowing the researcher to retain their data for analysis (in line with the British Psychological Society's guidelines (2009), participants were informed of the use of video cameras at the point of recruitment and their permission for being video recorded was sought on both consent forms, before and after the experiment). The experiment was approved by the local ethics committee.

Table 2

Overview of mean ratings for each of the scaled (1-7) questions from the addressee-behaviour-questionnaire.

\begin{tabular}{llllllll}
\hline Q1 & Q2 & Q3 & Q4 & Q5a & Q5b & Q5c & Overall mean \\
\hline 6.71 & 6.18 & 6.25 & 6.04 & 5.68 & 5.71 & 5.64 & 6.03 \\
\hline
\end{tabular}




\subsection{Data analysis}

\subsubsection{Speech transcription and word count}

Descriptions of the four target scenes (see section 2.2.2) were identified in the narratives from all participants, based on the notion of 'ideational units' (Butterworth, 1975; Holler and Beattie, 2002; Holler and Wilkin, 2009). This means that there were two units of which the words were counted for one target event (i.e., description of the same event before and after feedback). The respective speech was transcribed verbatim and the words relating to each of the target events were counted for each participant. This included all fillers and discourse markers ('like', 'you know'), but excluded 'ums' and 'uhs' (as these are non-lexical items and do not contain any semantic information themselves). Compound words (e.g., can't) were counted as two words. Words constituting self-repairs were included in the word count except for words which speakers left incomplete due to self- or other-interruptions. Note that not all of the participants received word counts for each of the four event descriptions, firstly, because some participants omitted some of the target events in their narrations, and, secondly, because sometimes no addressee feedback was delivered for a certain event (section 2.2.2). Also, in a few cases more than one reference to a particular aspect of an event was made before or after feedback; in those cases the first reference was considered in the analysis.

\subsubsection{Co-speech gesture coding}

Gestures were coded in two steps. Firstly, we identified all representational and non-representational gestures (excluding any movements which are not part of the speaker's communicative effort, such as self- and object-adaptors, Ekman and Friesen, 1969; Kendon, 1985). With respect to existing gesture classification schemes, we defined representational gestures as comprising iconic, metaphoric and deictic gestures (Alibali et al., 2001; McNeill, 1992) and non-representational gestures as comprising of those that do not primarily serve to depict semantic information but which fulfil pragmatic and interactive functions instead (Bavelas, 1994; Kendon, 2004; McNeill, 1992). Gestures of these two kinds (representational and nonrepresentational) were separated according to whether they occurred with the relevant speech units preceding the addressee's feedback or after the feedback. For each of the four target scenes we then calculated the gesture rate (rather than simple gesture frequencies, to account for differences in the amount of speech produced before and after feedback and for the individual scenes) by dividing the number of gestures by the respective number of words. This resulted in eight gesture rates per participant, two (representational and non-representational) for each of the four target scenes, that is, for the ideational units relating to each scene before and after feedback (as well as an overall gesture rate by collapsing the data across the scenes).

Secondly, amongst the representational gestures, we identified corresponding gesture pairs, that is, gestures that referred to the same semantic aspect of the target event both before and after addressee feedback (all of these gestures were iconic gestures, with one exception, which consisted of a gesture changing from an iconic to a deictic one). For example, a speaker might have referred to Scene 1 with the words 'and a car comes hurtling past and almost runs him over', accompanied by the right hand moving quickly from the right periphery of gesture space towards the left, representing the moving car; if this speaker referred to the same event in response to the addressee's feedback ('how close does it get to the man?') with the words 'rather close actually, the car almost touches him' accompanied by the right hand moving again from right to left but this time brushing past the speaker's left hand which is held flat against the own body, then these two gestures would be considered corresponding gestures.

Each of these corresponding gesture pairs was coded on four qualitative dimensions.

2.4.2.1. Gesture precision. We compared which of the two gestures of a pair was carried out with more movement precision (Gerwing and Bavelas, 2004), mainly determined through the degree of tension in the hands, arms and/or fingers (i.e., imprecise gestural movements tend to look more 'sloppy'). In the case of character-viewpoint gestures (section 2.4.2.4), including full body reenactments, weaker, or less extreme, movements were considered as less precise. Essentially, this dimension can be conceived of as the gestural equivalent of more precise verbal articulation. The coding categories for this dimension were defined as (a) less communicative = less precision; (b) same = no difference in precision; (c) more communicative $=$ more precision .

2.4.2.2. Gesture size. We judged which of the two gestures of a pair was larger based on their span (Beattie and Shovelton, 2005), i.e., how much of the speaker's gesture space (McNeill, 1992) the gestural movement occupied (in terms of the diameter of the 2-dimensionally plotted gesture movement at its widest point). The coding categories were here defined as (a) less communicative = smaller span; (b) same = no difference in span; (c) more communicative = bigger span.

2.4.2.3. Gesture space. We compared gesture pairs with respect to which of the two gestures was performed in more visually prominent areas of gesture space (Streeck, 1994), defined as either higher in gesture space, more central areas of gesture space, or both. Thus, there were two different aspects to consider here, and some gestures differed only in terms of height, others only in terms of how centrally they were performed. However, if a gesture pair differed in both respects, let's say the gesture after feedback was performed more centrally and higher in gesture space, this was not scored twice. Instead, the gesture was still classed as 'more communicative', just like a gesture after feedback that was either higher or more centrally performed while not differing on the respective other dimension (we thus applied a conservative measure which, if anything, 
might have underestimated the degree to which the gestures were recipient designed on this dimension). Further, when the gesture before feedback was, for example, more central than the gesture after, but the latter was performed higher in gesture space, then the two were considered to 'cancel each other out' and neither of the two gestures was considered as being more communicative than the other (i.e., they were coded as 'same').

In addition to these purely form-based criteria, for those cases where the gesture changed to being performed lower in gesture space after feedback, we considered the content of the target scene to determine whether the observed change was meaningful. For example, with regard to Scene 4, before feedback, some speakers referred to the children crouching down behind some grass with a gesture performed at about chest height moving slightly downwards, but representing the same event much lower at about the height of their lap after feedback. In this case, it would have been incorrect to conclude that the gesture after feedback was less communicative because it was performed lower down in gesture space, and the use of the lower gesture space here served the clearer communication of the event (an event that happened near the ground in physical space). However, in these cases speakers usually added more visual emphasis to the movement, moved their hand further forwards into shared gesture space (Özyürek, 2002), or to the side (in a way that made the gesture an ostensive object of joint visual examination, or, in another case, so that the table would not obstruct the view) to obtain visual prominence despite the lower location. Another case in which the purely form based criteria did not necessarily provide an accurate reflection of the degree of communicativeness for this category was when one speaker 'zoomed out' in terms of perspective to provide more accurate spatial information. When referring to Scene 6, this speaker used the coffee table placed between herself and the addressee to sketch out the spatial layout of the barn, the grass the children hide behind and so forth. Due to the low height of the coffee table, these gestures were performed lower in gesture space after feedback. However, they were performed in shared gesture space and more easily visually accessible from a (joint) bird's view (which clarified the spatial relations between the individual elements of the scene) and thus made the gestures visually prominent nevertheless. Although such exceptions were made in only a small number of cases, they have been flagged up in section 3 for maximum clarity (Table 4). The coding categories for gesture space were defined as follows: (a) less communicative = less central/lower gesture space; (b) same = no difference in spatial location; (c) more communicative $=$ more central/higher gesture space, plus the two exceptions described above.

2.4.2.4. Gesture viewpoint. We used McNeill's (1992) definition of character, observer and dual viewpoint to categorise the gestures as our starting point. According to McNeill, observer viewpoint (ovpt) gestures are characterized by the speaker's hands representing characters from the event as wholes. That is, the speaker's body is not immersed in the fictive space he or she is creating. Ovpt gestures thus incorporate a distance between narrator and the event. Character viewpoint (cvpt) gestures, on the other hand, are defined by the speaker taking on the role of the character. He or she is thus immersed in the fictive space around them and thus part of the depicted scene. His or her hands and arms represent the character's hands and arms (and sometimes also the head, torso and legs/feet) and depict the activities of these extremities. In addition, we identified cases in which the speaker also took on the perspective of the character by using his or her body as a reference point but without the hands and arms (or other extremities) carrying out the action the character performed (Holler and Beattie, 2003a). For example, consider a speaker using their right hand, moving from right to left through the central gesture space, to depict a car driving past (pure ovpt), as compared to the same gesture but this time sliding the right hand past the speaker's tummy to represent the distance (or lack thereof) between the car and the man (ovpt + cvpt perspective/reference point). In addition, there were cases in which the speaker did not just use their own body as a spatial reference point (representing the character's body) but where s/he embodied the actual actions of that character (i.e., a gesture with a character viewpoint rather than just a character perspective/reference point). Imagine the same gesture described above, but this time combined with the speaker moving their own torso backwards to depict the man stepping out of the way of the car (ovpt $+\mathrm{cvpt}$ ). This was termed a 'dual viewpoint' (dvpt) (McNeill, 1992). To summarise these differences into coding categories, we assumed (based on previous research, Beattie and Shovelton, 2002) that cvpt gestures are more communicative than ovpt gestures, and also that ovpt gestures plus additional cvpt components contained more information and thus are more communicative than pure ovpt gestures, with dvpt gestures being the most communicative. We used these gradations to classify any change in viewpoint (and perspective) as a change towards a more or less communicative gesture, thus arriving at three coding categories labeled 'less communicative', 'same', 'more communicative'.

\subsubsection{Inter-rater reliability}

Inter-rater reliability was obtained for all four gesture measures and resulted in substantial agreement (Landis and Koch, 1977 ) in each case (weighted Cohen's Kappa = .74 for 'gesture size', .75 as the average for both 'gesture space' measures, and .75 for 'gesture viewpoint'), with the exception of 'gesture precision', which obtained a result of .53. However, we examined the underlying pattern of divergence and a systematic difference was found; the second rater tended to judge gestures after feedback more often as more or less precise (in particular the latter) when the first (main) rater had judged the two gestures from a gesture pair as being of same precision. This reassured us that the judgements made by the first rater, who also coded the remainder of the dataset, were not biased towards the direction of the experimental prediction (on the contrary, there was not a single case in which the first rater judged a gesture after feedback to be more precise when the second rater did not). If anything, the first rater's judgements were more conservative here. So, on the whole, the coding for 'precision' showed moderate agreement (Landis and Koch, 1977); however, if we collapse the categories 'same' and 'less precise', which we treated here both as capturing gestures that are not recipient designed in terms of precision (i.e., they constitute 
essentially one category which is compared against gestures classed as being more precise after feedback), then agreement is substantial for this variable, too $(K=.78)$.

\subsubsection{Deixis in concurrent modalities}

We analysed deictic markers in speech and in gaze occurring together with the corresponding gestures before and after feedback. To code deictic markers in speech, we identified all demonstratives requiring a gesture complement (e.g., 'it came this close', 'the barn was here and the kids were here', 'it was about that high').

To code deixis in gaze, we identified all instances in which speakers shifted their gaze towards one of their gestures (irrespective of the duration of the gaze shift or at which point during the gesture they did so). In a few cases it was not possible to unambiguously identify gaze direction and those cases have been excluded from the analysis (12/100).

\section{Results}

For the statistical analyses reported below, we used Shapiro-Wilk tests to check for normality of the data and thus for our decision to use parametric tests or their non-parametric equivalents. An alpha level of .05 was used throughout. All tests reported are two-tailed.

Note that the $N$ for the individual comparisons varies, especially for the overall rate of gestures compared to the separate analyses for representational and non-representational gestures. The reason for this is that, if, for example, a speaker's overall gesture rate was zero after feedback then this data point would be included in the analysis of the overall gesture rate, but obviously not in following analyses categorising gestures as representational or non-representational. Further, note that the $N$ can differ for the analysis on gesture rates for the individual target scenes due to individual speakers sometimes not having referred to a scene or the corresponding feedback not having been delivered by the addressee (section 2.2 .2 ).

\subsection{Gesture rate before and after feedback}

First, we calculated the overall gesture rate before and after feedback. This analysis revealed that although speakers gestured at a numerically slightly higher rate before feedback $(M=.17, S D=.04)$ than after feedback $(M=.15, S D=.05)$, this difference was not statistically significant, $t(27)=1.49, p=.15$.

As a second step, we analysed gesture rate separately for representational and non-representational gestures. A Wilcoxon Signed Ranks test showed that the rate of representational gestures before feedback (Median $=.94$, Range $=.25)$ was not significantly different from that after feedback (Median $=1.0$, Range $=.50$ ), $z=1.29, N$-ties $=20, p=.20$. Likewise, the rate of non-representational gestures was also found not to be significantly different before (Median $=.06$, Range $=.25)$ and after feedback (Median $=.00$, Range $=.05), z=1.29, N$-ties $=20, p=.20$.

Following this, we analysed the gesture rates before and after feedback for the individual feedback types (i.e., in response to the four different types of feedback delivered by the addressee). This analysis showed, again, that speakers' gesture rate before and after feedback did not differ significantly for any of the feedback types, except for the last scripted question (i.e., seeking confirmation that understanding is correct); here, speakers used gestures at a significantly lower rate after feedback (see Table 3).

Representational and non-representational gestures were also analysed separately for each of the four feedback types. However, none of these comparisons showed significant differences.

\subsection{Qualitative nature of gestures before and after feedback}

For the form-based analysis, all corresponding gesture pairs were identified for the four target events (see section 2) resulting in 100 pairs of gestures (produced by 25 different participants) suitable for comparison. Out of these, seventy-six gestures (76\%) were recipient-designed in terms of having been judged as more communicative on one or more of the four qualitative dimensions we compared systematically (irrespective of how they were judged on the respective other dimensions). A Wilcoxon Signed Ranks test showed that the number of gestures judged to be more communicative on one or more of the four dimensions after feedback (Median $=3$, Range $=6$ ) was significantly larger than the number of gestures classed as not having changed in communicativeness, or that changed towards being less communicative, on any of the four dimensions after feedback (Median = , Range $=3$ ), $z=4.22, N$-ties $=23, p=.0001$.

\section{Table 3}

Overview of measures of central tendency, dispersion and statistical tests for each of the four feedback types (based on data combining representational and non-representational gestures).

\begin{tabular}{|c|c|c|c|}
\hline & Median (Range) before feedback & Median (Range) after feedback & Statistical test \\
\hline Feedback 1 & $.17(.08)^{\mathrm{a}}$ & $.16(.08)^{\mathrm{a}}$ & $t(24)=.48, p=.64, \mathrm{~ns}$ \\
\hline Feedback 2 & $.15(.25)$ & $.13(.21)$ & $z=0.11, N=18, p=.91, \mathrm{~ns}$ \\
\hline Feedback 3 & $.16(.20)$ & $.15(.25)$ & $z=0.34, N=20, p=.74, \mathrm{~ns}$ \\
\hline Feedback 4 & $.19(.22)$ & $.15(.50)$ & $z=2.08, N=28, p=.04$, sig \\
\hline
\end{tabular}

\footnotetext{
a Cells show means and standard deviations.
} 
In addition, a second Wilcoxon Signed Ranks test revealed that the number of gestures that was judged to be more communicative on one or more of the four dimensions after feedback (Median $=3$, Range $=6$ ) was significantly larger than the number of gestures judged to be more communicative on one or more of the four dimensions before feedback $($ Median $=1$, Range $=7$ ), $z=3.17, N$-ties $=23, p=.002$.

In a second step, we did not consider change towards increased communicativeness on individual dimensions irrespective of the respective other dimensions, but we considered the gestures in their entirety. That is, we allocated all gestures of a pair that occurred after feedback to one of three categories: (1) More communicative gestures, which comprised all those gestures that changed towards being more communicative overall, i.e., they changed into the predicted directions on at least one of the four dimensions and remained the same on all remaining ones (or, if they did show a decrease in communicativeness on any of the dimensions, the number of these was lower than the number of dimensions on which they changed towards being more communicative, i.e., the gesture became more communicative overall); (2) Less communicative gestures, which comprised all those gestures that changed towards being less communicative on one or more of the four dimensions and remained the same on all remaining dimensions (or, if they did show an increase in communicativeness on any of the dimensions, the number of these was lower than the number of dimensions on which they changed towards being less communicative, i.e., the gesture became less communicative overall); (3) Gestures that remained as communicative after feedback as before, which comprised all those gestures that were judged to be the same on all of the four dimensions before and after feedback, as well as those that changed towards being more communicative on one (or more) dimension(s), as well as towards being less communicative on exactly the same number of dimensions.

This analysis resulted in 60 gestures that were classed as more communicative after feedback, 28 gestures that were classed as less communicative after feedback, and 12 gestures that were classed as remaining unchanged in terms of their communicativeness. A Friedman test showed that the frequencies in these three categories differed significantly, $X^{2}(2)=16.83, p=.0001$. Wilcoxon Signed Ranks tests used to investigating the differences between the individual groups showed that significantly more gestures changed towards being more communicative (Median $=2.00$, Range $=6.00$ ) than less communicative (Median $=1.00$, Range $=4.00$ ), $z=2.88, N$-ties $=22, p=.004-$ in other words, gestures were judged to be more communicative after feedback than before. Also, significantly more gestures changed towards being more communicative than stayed the same (Median $=0.00$, Range $=2.00$ ), $z=3.64, N$-ties $=24, p=.0001$. Also, if we consider exclusively those gestures that were judged to be more communicative on one or more dimension and stayed the same on all remaining dimensions (52 gestures; Median $=2$, Range $=5$ ) and compare them to the number of gestures that were judged to be less communicative on one or more dimension and stayed the same on all remaining ones (18 gestures; Median = 1 , Range $=4$ ), we, again, find that gestures are more communicative after feedback than before, $z=3.17, N$-ties $=23, p=.002$.

Further, Table 4 provides an overview of the number of gestures that remained unchanged, changed towards being less communicative, or changed in the expected direction, split up by the individual dimensions. This overview reveals that about half of the gestures changed in the predicted directions for each of the three measures precision, size and space, with more than twice as many gestures being more communicative after feedback than before feedback (by definition, those gestures reported as less communicative were more communicative before feedback) for each of these three measures.

What is evident is that gesture viewpoint stands out as the one dimension with comparatively little change.

In addition, from Table 5 we can see that the majority of gestures were judged to be more communicative on one, two or three of the four qualitative dimensions under investigation here, with only very few gestures showing evidence of recipient design on all four dimensions simultaneously.

In addition to the four dimensions investigated here, speakers' gestures also showed evidence of being recipient-designed in terms of how they semantically represented the target events before and after feedback. For example, with respect to

Table 4

Frequencies for the four qualitative dimensions in terms of no change, change in the direction opposite to prediction, and predicted direction of change.

\begin{tabular}{llll}
\hline & Same & Less communicative & More communicative \\
\hline Precision & 48 & 12 & 40 \\
Size & 27 & 23 & 50 \\
Space & 29 & 21 & $50^{\text {a }}$ \\
Viewpoint & 71 & 11 & 18 \\
\hline
\end{tabular}

a $N B$ : 8 of these gestures were more communicative in lower space (see section 2.4.2.3).

Table 5

Overview of number of the four dimensions on which gestures were recipient designed/showed increased communicativeness.

\begin{tabular}{lr}
\hline Number of dimensions & Number of gestures \\
\hline 1 & 26 \\
2 & 23 \\
3 & 22 \\
4 & 5 \\
\hline
\end{tabular}


Scene 1, speakers tended to depict the movement of the car with simple right to left movements of the right hand, which in several cases brushed 'quite carelessly' against the speaker's body to show that the car got close to the man, without gesturally focusing on the exact distance between car and man (and the car did in fact not get in direct contact with the man). In response to the addressee's enquiry how close the car gets to the man, speakers tended to alter their representations by depicting a clear spatial distance between the hand representing the moving car and their own body, thus adapting the semantic focus of the gestural depiction to tailor it to the addressee's informational requirements. With regard to Scene 2 , the representation of the boys jumping off the top layer of the barn often involved the hand moving from right to left (or vice versa) in an arch like motion. In response to the question of whether the children jumped out of the barn, the gestural depiction following the feedback traced the path vertically (rather than horizontally or diagonally), thus clarifying that the movement occurred from one location in the barn to a location directly below, i.e., still within the barn. Regarding Scene 3, speakers tended to provide an overall fuller representation of what the boy was doing (in response to the question 'he did what?'), for example by representing not only part of the window frame and its location in front of the boy's head (by holding clasped hands below the chin) but also the fact that the boy stuck his head out through this window frame (by adding a head movement to the previous gesture). For Scene 4, speakers tended to depict a general movement path from left to right (or the other way round) to represent general movement outwards (sometimes combined with bobbing movements of the hand to illustrate that several entities were moving). After feedback requesting confirmation whether the children ran out of the barn and hid, speakers often traced the movement path more explicitly, depicting the exact route along which the children were running (out and around the barn, behind some crops). The adjusted gestural semantic representations of the target events are a reflection of the speaker's re-conceptualisation and 're-packaging' of elements of the target scenes tapped by the respective addressee's feedback.

Many gestures were recipient-designed in this way in response to addressee feedback in addition to being more communicative on one or more of the four dimensions. However, ten gestures were recipient-designed exclusively in terms of how depiction of semantic content was tailored to the addressee's feedback, but not on any of the four dimensions. This means that, in total, 86 gesture pairs (86\%) were recipient-designed in some way in response to addressee feedback when taking into account the four qualitative dimensions as well as any additional semantic adjustments (recipient-designed: Median = 3, Range = 6; not recipient-designed: Median $=0$, Range $=3$ ), $z=4.32, N$-ties $=24, p=.0001$.

\subsection{Indexicality in speech and gaze}

Elements of deixis concurrent with the corresponding gestures were evaluated for two modalities, speech and gaze. Analysis of the speech revealed that speakers included significantly more deictic elements (such as demonstratives) in their verbal utterance that referred explicitly to the gesture after feedback (Median $=0.00$, Range $=3.00$ ) than before feedback (Median $=0.00$, Range $=0.00$ ), $z=2.12, N$-ties $=5, p=.034$.

For the analysis of gaze, we excluded the data from two of the 25 participants for whom we identified corresponding gestures as their gaze direction could not be unequivocally identified. The same concerned nine corresponding gesture pairs from other participants. The analysis on those where gaze direction could be identified showed that speakers focused their gaze significantly more often on their gestures after feedback (Median $=1$, Range $=4$ ) than before feedback $($ Median $=0$, Range $=4), z=2.23, N$-ties $=11, p=.026$.

\section{Discussion}

The present analyses were aimed to reveal whether, and if so how, co-speech gestures are affected by addressee feedback in dyadic face-to-face interactions. This concerned their proportional frequency but also their qualitative nature.

We found, firstly, that gesture rate did not differ significantly before and after addressee feedback, which suggests that speakers continue to draw on the gestural modality when responding to addressee feedback, despite communicating the respective message for a second time. We found this to be the case for both representational and non-representational gestures. Moreover, we found that this pattern holds for addressee questions requiring different speaker responses: speakers continued to use gesture to the same extent before and after feedback when they were required to provide more detail or clarity regarding a specific semantic aspect of the event they were talking about as well as when their addressee signalled more general non-understanding of what was said about the event as a whole. Likewise, speakers gestured at the same rate before and after feedback when this feedback indicated that the addressee had understood aspects of the described event incorrectly.

The only exception to this pattern was that of addressee feedback signalling correct understanding of the event the speaker intended to convey. In this case, speakers' communicative intent will have been different (confirming that how the event was understood is correct, rather than trying to repair or elaborate). Although most speakers did not merely respond with 'yes' but repeated aspects of the event ('yes, they all run out', 'yes, they all manage to get out and hide'), they accompanied these utterances with gesture to a significantly lesser extent than they did with their initial description of this event. Thus, the analysis suggests that it may not be addressee feedback per se, and the potentially greater interactional involvement that results from it, that encourages speakers to respond with speech-gesture utterances. Instead, the specific content of the addressee's feedback and its direct link to the speaker's communicative intent appears to be crucial. However, we have to be tentative with our conclusions here, the reason being that only one type of feedback elicited a confirmatory 
response, and this feedback was always paired with the same event description. Although the event structure (i.e., two action elements, children running out of a building, then hiding in the grass) and its standard description were of the same type as some of the other event descriptions, we currently cannot exclude the possibility that something related to the content of the event itself may have led to the difference in gestural response after feedback.

While the overall rate of gesturing before and after feedback did not differ, the qualitative nature of the gestures did. Here, we selected four dimensions for which change in a particular direction has been linked to increased communicativeness in the existing literature. This showed that the significant majority of the gestures included in this second analysis showed evidence of increased communicativeness (on one or more of the selected dimensions), and were more communicative overall, in response to addressee feedback, a clear signs of recipient design. When considering the four qualitative dimensions individually, it became evident that there does not appear to be one predominant pattern characterizing the change in gesture form. Rather, gestures in response to feedback appeared to be more precise, bigger, or more visually prominent to roughly equal extent (however, most convincing is the change towards bigger and more visually prominent gestures, as gestures were twice more likely to increase in communicativeness than they were to stay the same or change towards being less communicative regarding these two variables). Furthermore, an equal number of gestures changed towards being more communicative on one, two or three of the four dimensions. This supports Kendon's $(1985,2004)$ idea that the gesture-speech system is characterized by flexibility so that the form of both the verbal and the gestural component of the utterance can be adapted to meet current communicational demands.

The situation looked different for the dimension of gesture viewpoint. Here, we found that the large majority of gestures remained unchanged. One difference between viewpoint and the remaining three qualitative dimensions examined here is that the former is more directly tied to the semantic information depicted by the gestures. That is, when a speaker changes the location of their gesture in space, performs a larger gesture or a more precise one, this can affect the semantic information depicted by the gesture (e.g., the depiction of height in the case of a higher location in gesture space, the depiction of size in the case of a larger gesture). However, it is also possible that the semantic information represented remains unaffected (e.g., a round shape remains a round shape no matter where in gesture space it is depicted or how large the gesture is). Changes in gesture viewpoint/perspective, on the other hand, always affect the semantic content of the gestural representation and the gesture's semantic focus. This qualitative dimension is thus more influenced, and in some way more restricted, by the underlying event structure and thus may be less susceptible to change due to interactional influences. However, this is a speculative explanation at most and requires further research.

In sum, when addressee feedback does signal a problem with understanding, then speakers do not only draw upon gesture with the same proportional frequency before and after addressee feedback, but they also adjust their gestural representations to make the utterance better understandable the second time round. Less than a quarter of the gestures in our corpus of corresponding gestures did not show any sign of increased communicativeness on one of the four dimensions analysed here, and just fourteen percent showed no clear sign of recipient design on these dimensions at all, not even by adjusting the semantic information depicted in response to the addressee's feedback. Descriptive analyses have provided evidence for both gestural repetition as well as change in response to addressee feedback (Kendon, 2004). By investigating a larger corpus of gestures systematically we are able to confirm the occurrence of both types of gestural responses, but we have obtained much stronger evidence for gestural change.

In addition, two further analyses corroborated the finding that speakers consider gesture an important communicative resource in responding to addressees' expressed lack of understanding. Both verbal deixis (it's this big) and speakers' gaze directed at their own gestures are clear markers of the gesture's communicative value (Streeck, 1993; Goodwin, 1986). Both occurred significantly more frequently in response to addressee feedback than before.

The findings from the present study build on earlier work which has shown that the presence of an addressee who can see the speaker's gestures influences the speaker's gesture rate (Alibali et al., 2001; Bavelas et al., 2002, 2008; Mol et al., 2009) as well as the semantic interaction of gesture and speech (Bavelas et al., 2002, 2008). Here we go beyond this effect of copresence and visibility, however, by focusing on the interactive process between speaker and addressee. In addition to affecting speakers' gestures by being present and able to perceive the gestures, addressees appear to influence speakers' gestures through their own responses, especially if they seem to be struggling to understand what is being conveyed.

Jacobs and Garnham (2007) showed that speakers gesture at a higher rate when talking to attentive addressees than distracted ones. Here, we build on this finding by zooming into the very process of addressee feedback. Rather than comparing more or less feedback (the addressee remained attentive throughout), we focused on different types of feedback (signalling understanding and non-understanding) and the effects on both gesture rate and the qualitative nature of speakers' gestures. The present study thus advances our understanding of addressee behaviour on speakers' use of co-speech gestures.

Further, the present study complements work by Bavelas et al. $(1992,1995)$ which has identified a type of gesture called 'interactive gestures'. These gestures are used by speakers to address and involve the recipient in the interaction in a number of different ways (such as by seeking feedback or agreement). This is evidenced by the fact that addressees reliably issue appropriate responses to utterances delivered with interactive gestures (Bavelas et al., 1995). The present study represents the other side of this coin; whereas Bavelas et al.'s $(1992,1995)$ work explored how speakers use gesture to elicit addressee responses, here we focused on addressees eliciting gestural responses. The present study thus complements this earlier research nicely, and together they provide us with a fuller understanding of the role of co-speech gestures in the process of face-to-face dyadic social interaction. In particular, the findings further our insights into the pragmatics of co-speech 
gestures. The findings thus lend support to theories of gesture production postulating that co-speech gestures are tightly linked to the speaker's communicative intent, with gestures benefitting social and communicative processes in interaction (Bavelas and Chovil, 2000; Clark, 1996; Kendon, 2004). Furthermore, the findings may be of relevance in the context of communication disorders such as aphasia, as, here, language can be more affected than gesture, with the latter fulfilling a compensatory function (Feyereisen, 1983; Goodwin, 2000). It may be especially important to attend to those patients' gestures in responses to addressee feedback as aphasics can be impaired in their verbal ability to provide such responses (e.g., reduced use of expansions in response to feedback; Rezania et al., 1989).

Through systematic comparisons, the present study has illuminated the so far rather neglected topic of how addressee feedback affects gesture use in dyadic interaction. However, it also has a number of limitations. Firstly, a confederate took on the role of the addressee and delivered a range of scripted feedback behaviours at pre-determined points in the narratives. In order to limit the possibility that the confederate's behaviour could have been experienced as unnatural by the speakers, we eliminated those trials from our analysis which two judges classed as noticeably different from the other trials in that the confederate's scripted feedback behaviour either differed from the scripted form or appeared unnatural in the manner in which it was delivered. Further, the questionnaires speakers completed at the end of the experiment revealed that they experienced their addressee as cooperative, interested, attentive and helpful in their communicative behaviour, which was the case throughout (even on the later trials) thus ruling out the potential criticism of confederate fatigue. However, our confederate did show some variation in her behaviour in terms of whether she used a gesture or not, and aspects of behaviour such as facial expressions could not be controlled in this setting. While the variation in gesture did not appear to have had any effect on the present data (see section 2), it is an issue that could be explored more systematically in future studies. In terms of facial expressions, it could be assumed that speakers may feel more encouraged to provide good feedback responses for smiling/friendly looking addressees than for non-smiling ones/less friendly looking addressees, for example. Since we used a within-participant design, such influences should be minimal; however, theoretically, it could have played a role in cases in which the addressee might have adopted a different mood/facial expression while the speaker provided the response to her feedback compared to when s/he provided the first account of the respective scene. To what extent co-speech gestures are sensitive to such subtle changes in affiliative expressions remains to be explored in future studies.

Another point of limitation is the type of feedback that we employed. In spontaneous interaction, the range of feedback behaviours delivered by addressees is wider of course, including, for example, contradictions, agreements, expressions of surprise or doubt and so forth (and nonverbal signals such as facial expressions and prosody play a crucial role here, too). Although the feedback delivered by the confederate in the present study was based on the kind of feedback observed in spontaneous interactions (and spontaneous feedback was delivered in addition to the scripted responses), it is not clear to what extent our findings can be generalised to other kinds of feedback that we did not systematically manipulate here. Similarly, the present research focuses on native speakers of British English only and speakers from other cultures and of other languages may, of course, behave differently in response to addressee feedback. And even amongst speakers of one language, the influence of the social context may play an important role, such as group size (here we focused on dyadic interaction only), the content of talk (e.g., narrative of concrete actions and events versus emotion talk), as well as the speakers' communicative intent (e.g., to give the other person a good understanding of a narrative plotline versus to convince or persuade someone). We know remarkably little about the effects of all these variables on gestural behaviour and future research is urgently needed to complete our insights in these respects.

Finally, we have here focused on a limited range of variables capturing change in speakers' gestural behaviour in response to addressee feedback. Our choice of variables was motivated by previous literature that had established a link between speaker's communicative intent, gesture and recipient design. It is of course possible that gestures show signs of recipient design in response to addressee feedback on a number of additional variables, too. However, with regard to the type of talk we focused on here, the variables we chose seemed to capture the gestural change we observed, in addition to the semantic changes we briefly addressed, rather well.

\subsection{Conclusion}

In sum, the present study has provided us with a first glimpse of speakers' gestural behaviour in response to addressee feedback. We were able to show not only that speakers deem gesture to be an important modality required for responding to this feedback but also that the majority of gestures show signs of change which contribute to making the speaker's message more communicative and thus suitable for addressing the recipient's informational requirements.

\section{Acknowledgements}

We would like to thank the Economic and Social Research Council (UK) for funding this research through a grant awarded to the first author (RES-061-23-0135), Kate Webster and Valentina Gratton for their help with data collection, as well as the participants who took part in this research. Further, we would like to thank Spencer Kelly and Stefan Kopp for useful feedback in discussions of this research, as well as two anonymous reviewers for their helpful comments on an earlier version of this manuscript. 


\section{References}

Alibali, Martha W., Heath, Dana C., Myers, Heather J., 2001. Effects of visibility between speaker and listener on gesture production: some gestures are meant to be seen. Journal of Memory and Language $44,1-20$.

Allwood, Jens, Ahlsén, Elisabeth, 1999. Learning how to manage communication, with special reference to the acquisition of linguistic feedback. Journal of Pragmatics 31, 1353-1389.

Bavelas, Janet B., 1994. Gestures as part of speech: methodological implications. Research on Language and Social Interaction 27, $201-221$.

Bavelas, Janet B., Chovil, Nicole, 2000. Visible acts of meaning: an integrated message model of language in face-to-face dialogue. Journal of Language and Social Psychology 19, 163-194.

Bavelas, Janet B., Chovil, Nicole, Lawrie, Douglas A., Wade, Allan, 1992. Interactive gestures. Discourse Processes 15, 469-489.

Bavelas, Janet B., Chovil, Nicole, Coates, Linda, Roe, Lori, 1995. Gestures specialized for dialogue. Personality and Social Psychology Bulletin 21, $394-405$.

Bavelas, Janet B., Coates, Linda, Johnson, Trudy, 2000. Listeners as co-narrators. Journal of Personality and Social Psychology 79, 941-952.

Bavelas, Janet B., Kenwood, Christine, Johnson, Trudy, Phillips, Bruce, 2002. An experimental study of when and how speakers use gestures to communicate. Gesture 2, 1-18.

Bavelas, Janet B., Gerwing, Jennifer, Sutton, Chantelle, Prevost, Danielle, 2008. Gesturing on the telephone: independent effects of dialogue and visibility. Journal of Memory and Language 58, 495-520.

Beattie, Geoffrey, Aboudan, Rima, 1994. Gestures, pauses and speech: an experimental investigation of the effects of changing social context on their precise temporal relationship. Semiotica 99, 239-272.

Beattie, Geoffrey, Shovelton, Heather, 2002. An experimental investigation of some properties of individual iconic gestures that mediate their communicative power. British Journal of Psychology 93, 179-192.

Beattie, Geoffrey, Shovelton, Heather, 2005. Why the spontaneous images created by the hands during talk can help make TV advertisements more effective. British Journal of Psychology 96, 21-37.

British Psychological Society Code of Ethics \& Conduct, 2009. Published by the Ethics Committee of the British Psychological Society, Leicester, UK.

Butterworth, Brian, 1975. Hesitation and semantic planning in speech. Journal of Psycholinguistic Research 4, 75-87.

Clark, Herbert H., 1996. Using Language. Cambridge University Press, Cambridge.

Clark, Herbert H., Brennan, Susan A., 1991. Grounding in communication. In: Resnick, L.B., Levine, J.M., Teasley, S.D. (Eds.), Perspectives on Socially Shared Cognition. APA Books, Washington, pp. 127-149.

Clark, Herbert H., Krych, Meredith A., 2004. Speaking while monitoring addressees for understanding. Journal of Memory and Language 50, 62-81.

Clark, Herbert H., Schäfer, Edward F., 1987. Concealing one's meaning from overhearers. Journal of Memory and Language 26, $209-225$.

de Fornel, Michel, 1992. The return gesture: some remarks on context, inference, and iconic gesture. In: Auer, P., Di Luzio, A. (Eds.), The Contexualisation of Language. John Benjamins, Amsterdam, pp. 159-176.

De Ruiter, Jan Peter, Noordzij, Matthijs L., Newman-Norlund, Sarah E., Newman-Norlund, Roger D., Hagoort, Peter, Levinson, Stephen C., Toni, Ivan, 2010. Exploring the cognitive infrastructure of communication. Interaction Studies 11, 51-77.

Ekman, Paul, Friesen, Wallace V., 1969. The repertoire of nonverbal behavior: categories, origins, usage, and coding. Semiotica 1, 49-98.

Enfield, Nick, Kita, Sotaro, De Ruiter, Jan-Peter, 2007. Primary and secondary pragmatic functions of pointing gestures. Journal of Pragmatics 39, $1722-1741$.

Feyereisen, Pierre, 1983. Manual activity during speaking in aphasic subjects. International Journal of Psychology 18, 545-556.

Furuyama, Nobuhiro, 2000. Gestural interaction between the instructor and the learner in origami instruction. In: McNeill, D. (Ed.), Language and Gesture. Cambridge University Press, Cambridge, pp. 99-117.

Garfinkel, Harold, 1967. Studies in Ethnomethodology. Prentice-Hall, Englewood Cliffs, NJ.

Garrod, Simon, Anderson, Anne, 1987. Saying what you mean in dialogue: a study in conceptual and semantic co-ordination. Cognition 27, 181-218.

Gerwing, Jennifer, Bavelas, Janet, 2004. Linguistic influences on gesture's form. Gesture 4, 157-195.

Goodwin, Charles, 1986. Gesture as a resource for the organization of mutual orientation. Semiotica 62, 29-49.

Goodwin, Charles, 2000. Gesture, aphasia, and interaction. In: McNeill, D. (Ed.), Language and Gesture. Cambridge University Press, Cambridge, pp. 84-98.

Grice, H. Paul, 1975. Logic and conversation. In: Cole, P., Morgan, J. (Eds.), Syntax and Semantics. Academic Press, New York, pp. 41-58.

Gullberg, Marianne, Holmqvist, Kenneth, 1999. Keeping an eye on gestures: visual perception of gestures in face-to-face communication. Pragmatics and Cognition 7, 35-63.

Gullberg, Marianne, Holmqvist, Kenneth, 2006. What speakers do and what listeners look at: visual attention to gestures in human interaction live and on video. Pragmatics and Cognition 14, 53-82.

Holler, Judith, 2010. Speakers' use of interactive gestures to mark common ground. In: Kopp, S., Wachsmuth, I. (Eds.), Gesture in Embodied Communication and Human-Computer Interaction, 8th International Gesture Workshop, Bielefeld, Germany, 2009, Selected Revised Papers, Springer Verlag, Heidelberg, pp. 11-22.

Holler, J., Beattie, G., 2002. A micro-analytic investigation of how iconic gestures and speech represent core semantic features in talk. Semiotica 142, 31-69.

Holler, Judith, Beattie, Geoffrey, 2003a. How iconic gestures and speech interact in the representation of meaning: are both aspects really integral to the process? Semiotica $146,81-116$

Holler, Judith, Beattie, Geoffrey, 2003b. Pragmatic aspects of representational gestures: do speakers use them to clarify verbal ambiguity for the listener? Gesture 3, 127-154

Holler, J., Stevens, R., 2007. An experimental investigation into the effect of common ground on how speakers use gesture and speech to represent size information in referential communication. Journal of Language and Social Psychology 26, 4-27.

Holler, Judith, Wilkin, Katie, 2009. Communicating common ground: how mutually shared knowledge influences the representation of semantic information in speech and gesture in a narrative task. Language and Cognitive Processes 24, 267-289.

Holler, Judith, Wilkin, Katie, 2011. Co-speech gesture mimicry in the process of collaborative referring during face-to-face dialogue. Journal of Nonverbal Behavior 35, 133-153.

Hostetter, Autumn B., Alibali, Martha W., Sotaro, Kita, 2007. I see it in my hands' eye: representational gestures reflect conceptual demands. Language and Cognitive Processes 22, 313-336.

Jacobs, Naomi, Garnham, Alan, 2007. The role of conversational hand gestures in a narrative task. Journal of Memory and Language 56, $291-303$.

Kendon, Adam, 1985. Some uses of gesture. In: Tannen, D., Saville-Troike, M. (Eds.), Perspectives on Silence. Ablex, Norwood, New York, pp. $215-234$.

Kendon, Adam, 2004. Gesture: Visible Action as Utterance. Cambridge University Press, Cambridge.

Kimbara, Irene, 2006. On gestural mimicry. Gesture 6, 39-61.

Kimbara, Irene, 2008. Gesture form convergence in joint description. Journal of Nonverbal Behavior 32, 123-131.

Kita, S. (Ed.), 2003. Pointing: where language, culture, and cognition meet. Lawrence Erlbaum, Mahwah, NJ.

Kita, Sotaro, Davies, Thomas S., 2009. Competing conceptual representations trigger co-speech representational gestures. Language and Cognitive Processes 24, 761-775

Kopp, Stefan, Allwood, Jens, Ahlsen, Elisabeth, Grammer, Karl, Stocksmeier, Thorsten, 2008. Modeling embodied feedback in a virtual human. In: Wachsmuth, I., Knoblich, G. (Eds.), Modeling Communication with Robots and Virtual Humans, vol. 4930. Springer Verlag, Berlin, pp. 18-37.

Krauss, Robert M., Weinheimer, Sidney, 1966. Concurrent feedback, confirmation and the encoding of referents in verbal communication. Journal of Personality and Social Psychology 4, 343-346.

Kraut, Robert E, Lewis, Steven H., Swezey, Lawrence W., 1982. Listener responsiveness and the coordination of conversation. Journal of Personality and Social Psychology 43, 718-731.

Landis, J. Richard, Koch, Garry G., 1977. The measurement of observer agreement for categorical data. Biometrics 33, 159-174. 
McNeill, David, 1992. Hand and Mind. The University of Chicago Press, Chicago.

Mol, Lisette, Krahmer, Emiel, Maes, Alfons, Swerts, Marc, 2009. The communicative import of gestures: evidence from a comparative analysis of humanhuman and human-machine interactions. Gesture 9, 97-126.

Morsella, Ezequiel, Krauss, Robert M., 2004. The role of gestures in spatial working memory and speech. The American Journal of Psychology 117, 411-424.

Özyürek, Asli, 2002. Do speakers design their cospeech gestures for their addressees? The effects of addressee location on representational gestures. Journal of Memory and Language 46, 688-704.

Parrill, Fey, 2010. The hands are part of the package: gesture, common ground, and information packaging. In: Newman, J., Rice, S. (Eds.), Empirical and Experimental Methods in Cognitive/Functional Research. CSLI, Stanford, pp. 285-302.

Pine, Karen J., Gurney, Daniel, Fletcher, Ben, 2010. The semantic specificity hypothesis: when gestures do not depend upon the presence of a listener. Journal of Nonverbal Behaviour 34, 169-178.

Rezania, Keveh, Hambrecht, Georgia, Quist, Raymond, 1989. How do aphasic and normal speaking subjects restate a message in response to feedback? Journal of Communication Disorders 22, 13-21.

Sacks, Harvey, Schegloff, Emanuel A., Jefferson, Gail, 1974. A simplest systematics for the organization of turn-taking in conversation. Language 50, 696-735. Schegloff, E.A., 1982. Discourse as an interactional achievement: some uses of "uh huh" and other things that come between sentences. In: Tanne, D.

(Ed.), Georgetown University Round Table on Languages and Linguistics 1981. Georgetown University Press, Washington, D.C., pp. 71-93.

Schober, Michael F., Clark, H.H., 1989. Understanding by addressees and overhearers. Cognitive Psychology 21, 211-232.

Slama-Cazacu, Tatiana, 1976. Nonverbal components in message sequence: 'Mixed syntax'. In: McCormack, W.C., Wurm, S.A. (Eds.), Language and Man: Anthropological Issues. Mouton, The Hague, pp. 217-222.

Stivers, Tanya, Enfield, Nick J., 2010. A coding scheme for question-response sequences in conversation. Journal of Pragmatics 42, $2620-2626$.

Streeck, Jürgen, 1993. Gesture as communication I: its coordination with gaze and speech. Communication Monographs 60, 275-299.

Streeck, Jürgen, 1994. Gesture as communication II: the audience as co-author. Research on Language and Social Interaction 27, $239-267$.

Tabensky, Alexis, 2001. Gesture and speech rephrasings in conversation. Gesture 1, 213-235.

Yngve, Victor H., 1970. On getting a word in edgewise. Paper from the Sixth Regional Meeting of the Chicago Linguistics Society, pp. 567-577.

Dr. Judith Holler is Assistant Professor in Psychology at the University of Manchester (UK) and currently on secondment at the Max Planck Institute for Psycholinguistics, The Netherlands, as a research fellow. Her research interest is multimodal communication, with a focus on co-speech gestures. In particular, she is interested in how communicative intent and the pragmatics of conversation influence gesture use and the interplay between gesture and speech, as well as how social cues and context may modulate the perception and communicativeness of co-speech gestures and their integration with speech.

Ms. Katie Wilkin is a Research Assistant at the University on Manchester. She has completed several RA posts on language and communication related topics and currently working in the area of Clinical Psychology. 